\title{
Number and Necessity of Tests Performed In the Last Week of Life of a Cancer Patient
}

\begin{abstract}
Keywords: Necessary; Test; Terminal; Cancer
Abstract

Introduction: During the last few months of life patients often suffe from significant psychological and physical distress. Studies in the past have found that the amount of tests conducted on a patient tends to increase as death approaches. In many cases the tests performed on a patient in the last few months of life were unnecessary, causing needless discomfort. This study focuses on the number and necessity of tests conducted in the last week of life of a terminal cancer patient.

Methods: This study was conducted at Liverpool Hospital, a university teaching hospital located in New South Wales, Australia. Data from deceased cancer patients referred to palliative care for consultation was collected retrospectively from electronic medica records and patient clinical notes. A standard data extraction form was developed to collect patient characteristics, tests ordered, patient progress and symptoms over a period of 7 days up until death. Two palliative care specialists not involved with care of these patients then independently evaluated the need and outcome of each test. These specialists were aware that the patients had all died.
\end{abstract}

Results: 33 patients were included in the study over a period of 3 months. A total number of 1051 tests were conducted over a combined total of 231 in-hospital days. The average number of tests conducted during the last 7 days prior to death per patient was 31.85. On average $65.16 \%$ of tests conducted were assessed as unnecessary $28.80 \%$ neutral and $6.04 \%$ necessary per patient. The most frequent test category ordered was blood chemistry $(41.19 \%)$ followed by complete blood count (28.83\%) and coagulation (10.47\%) tests. In total, combined over all 33 patients, $69.27 \%$ of the tests were deemed unnecessary, $25.21 \%$ neutral and $5.52 \%$ necessary

Conclusion: End of life cancer patients are subjected to frequen and often unnecessary tests and investigations. Much improvemen can be made in hospital systems and clinician education in order to increase the quality of patient care. Our study prompts clinicians to evaluate the necessity and benefit of diagnostic tests conducted on end of life cancer patients before performing them and provides recommendations for improvement.

\section{Introduction}

During the last few months of life, patients often suffer from significant psychological and physical distress [1], and many are hospitalized prior to death. However within hospitals there exists an acute care culture consisting of tests and treatments to diagnose and treat any potentially reversible causes, and failure to recognize when further disease-modifying treatments have no benefit can potentially result in neglect of appropriate comfort measures [2], including discontinuation of unpleasant and unnecessary tests. The number of tests conducted on a patient tends to increase as death approaches. A study by Asola et al. found that laboratory and radiological tests conducted on patients with advanced breast cancer were respectively 2.4 and 1.4 times more frequent in the month prior to death compared to that of six months earlier, with almost half the total number of tests conducted in the last six months of life performed in the last two months [3]. Although appropriateness of a test is an

\section{Journal of}

Geriatrics and Palliative Care

Rachel WC NG ${ }^{1}$, Chi Eung Danforn LIM ${ }^{1 *}$, Nga Chong Lisa CHENG ${ }^{1}$, Rebecca STRUTT ${ }^{2}$, Jennifer WILTSHIRE $^{2}$, Maria CIGOLINI ${ }^{3}$ and Christopher ZASLAWSKI ${ }^{4}$

${ }^{1}$ South Western Sydney Clinical School, Faculty of Medicine, University of New South Wales, Australia

${ }^{2}$ Department of Palliative Medicine, Liverpool Hospital, Sydney, Australia

${ }^{3}$ Department of Palliative Medicine, Royal Prince Alfred Hospital, Sydney, Australia

${ }^{4}$ School of Medical and Molecular Biosciences, University of Technology Sydney, Australia

\section{Address for Correspondence}

Danforn Lim, Faculty of Medicine, University of New South Wales,PO Box 3256, Blakehurst, NSW 2221 Australia, Email: celim@unswalumni.com

Copyright: ( 2013 Rachel WC, et al. This is an open access article distributed under the Creative Commons Attribution License, which permits unrestricted use, distribution, and reproduction in any medium, provided the original work is properly cited.

Submission: 28 October 2013

Accepted: 18 December 2013

Published: 23 December 2013

Reviewed \& Approved by: Dr. Egidio Del Fabbro, Associate Professo in the Division of Hematology, Oncology and Palliative Care of the Department of Internal Medicine at the VCU School of Medicine, USA

independent measure from frequency of investigation [1], in many cases the tests performed on a patient in the last few months of life were unnecessary, causing needless discomfort. A study conducted by Toscani et al. found that even in patients whose deaths were highly expected, 57\% still received routine blood tests in the final 24 hours of life [4]. Subjecting terminal patients to medical interventions like 'routine' tests such as taking blood, nasogastric tubes or placing intravenous lines can be a traumatic experience to patients [5]. Although many studies have focussed on treatments performed in the end of life stages of cancer patients $[3,6]$, few have specifically examined the number and necessity of diagnostic tests conducted in the last days of life.

This study focuses on the number and necessity of tests conducted in the last week of life of an end of life cancer patient.

\section{Methods}

\section{Study setting}

The study was carried out at Liverpool Hospital, South Western Sydney, New South Wales, Australia.

\section{Patient inclusion criteria}

All patients with an established diagnosis of cancer who were admitted to Liverpool Hospital and referred to the Department of Palliative Care for consultation and who died between $1^{\text {st }}$ May 2012 and $31^{\text {st }}$ July 2012 were included in the study. All eligible patients must have been admitted into Liverpool Hospital for at least seven days during their final admission, deemed to be in terminal care and died during this admission. 
Citation: Rachel WC NG, Chi Eung Danforn LIM, Nga Chong Lisa CHENG, Rebecca STRUTT, Jennifer WILTSHIRE, et al. Number and Necessity of Tests Performed In the Last Week of Life of a Cancer Patient. J Geriatrics Palliative Care 2013;1(1): 5.

ISSN: 2373-1133

\section{Study design}

1.Definition of standards and rating system used to evaluate tests conducted agreed upon by investigators and palliative care specialists involved

2.Retrospective collection of data of eligible patients from electronic medical records and patient clinical notes

3.Evaluation and review of tests conducted by two clinically active palliative care specialists not involved in management of the patients included in this study

\section{Data collection}

A standard data extraction form was developed to collect the following pieces of information from the electronic medical records system and patient clinical records.

a)Patient information: including age, gender, date deceased, date of final admission and date referred to palliative care

b)Patient overall diagnosis and past medical history

c)Symptoms displayed and clinical progress of patient over the last seven days prior to death

d)Tests conducted in the last seven days prior to death: including date performed, test type, individual tests ordered and results

\section{Necessity of investigations}

Two clinically active palliative care specialists not involved with the care of the patients, individually evaluated each test for each patient, according to the standards (Table 1) agreed upon prior to commencement of study. The palliative care specialists were acted as assessors and were then assigned an appropriateness rating score of either: 1 - very inappropriate, 2 - inappropriate, 3 - neutral, 4 - appropriate or 5 - very appropriate. The two sets of scores were then averaged and divided into the three categories: Unnecessary (1 - 2), Neutral (2.5 - 3.5) and Necessary (4 - 5) according to the mean score. Cross examination of the scores were performed between the assessors. Should there be any discrepancy between the scores given by 2 assessors, a senior specialist of Palliative medicine would be sought to provide a third opinion and determine the final scores.

\section{Statistical analysis}

Simple quantitative data analysis of summarising measurements including mean, median, range and percentages were reported. The Statistical Package for the Social Sciences (SPSS) software version 17.0 was used for statistical analysis. Pearson's correlation test was conducted to examine nonparametric variable relationships. An interrater analysis using the Kappa statistic was used to determine the consistency between specialists.

\section{Results}

\section{Baseline characteristics of patients}

During the 3 month study period, a total of 33 cases met the inclusion criteria and were included in the study. Patients were admitted to hospital for a variety of reasons including dyspnoea, weakness and pain. 20 of the 33 cases were male and the mean age of the patients was 65.73 years. The mean length of final admission

\section{Table 1: Standards of diagnostic tests}

a) Diagnostic tests should only be performed to identify reversible causes of clinical deterioration and monitor current treatment regimes

b) Invasive tests should not be performed unless indicated

\begin{tabular}{|c|c|c|c|}
\hline Characteristics & Male & Female & Total \\
\hline Number & 20 & 13 & 33 \\
\hline Mean Age & 68.60 & 61.31 & 65.73 \\
\hline $\begin{array}{l}\text { Mean length of final admission } \\
\text { (Days) }\end{array}$ & 21.10 & 26.62 & 23.27 \\
\hline $\begin{array}{l}\text { Mean length of palliative care } \\
\text { referral during final admission } \\
\text { (Days) }\end{array}$ & 7.89 & 9.09 & 8.34 \\
\hline \multicolumn{4}{|l|}{ Cancer diagnosis } \\
\hline Breast & 0 & 2 & 2 \\
\hline Gastrointestinal & 2 & 2 & 4 \\
\hline Genitourinary & 5 & 0 & 5 \\
\hline Gynaecologic & 0 & 2 & 2 \\
\hline Hematologic & 3 & 2 & 5 \\
\hline Head and Neck & 2 & 1 & 3 \\
\hline Lung & 6 & 0 & 6 \\
\hline Neurologic & 0 & 1 & 1 \\
\hline Other & 0 & 1 & 1 \\
\hline Unknown Primary & 2 & 2 & 4 \\
\hline
\end{tabular}

and mean length of palliative care referral was 23.27 days and 8.34 days respectively. The most common primary cancer type was Lung cancer (Table 2).

\section{Number of tests}

A total number of 1051 tests were conducted over a combined total of 231 in-hospital days. The average number of tests conducted during the last seven days prior to death per patient was 31.85 . Pearson's correlation test showed a negative relationship between total number of tests and length of palliative care referral (Pearson Correlation coefficient $-0.442, p=0.016$ ). There was no statistically significant relationship between gender, age or total length of admission and total number of tests or proportion that were ranked unnecessary.

On average, there were a total of $5.79,6.30,5.91,4.33,4.88,2.94$ and 1.70 tests conducted on patients from day 1 to 7 in their last week of life respectively. There was a slight decreasing trend in the total number of tests conducted per day in the last 7 days prior to death with a similar trend in the number of tests ranked neutral and necessary (Figure 1).

\section{Necessity of tests}

On average $65.16 \%$ of tests conducted were ranked unnecessary, $28.80 \%$ neutral and $6.04 \%$ necessary per patient. The highest numbers of unnecessary tests were conducted in day 3 (4.18) and day 5 (3.85) of the last week of life. However, the proportion of tests ranked unnecessary increased over the seven day period with $94.64 \%$ of tests 
Citation: Rachel WC NG, Chi Eung Danforn LIM, Nga Chong Lisa CHENG, Rebecca STRUTT, Jennifer WILTSHIRE, et al. Number and Necessity of Tests Performed In the Last Week of Life of a Cancer Patient. J Geriatrics Palliative Care 2013;1(1): 5.

ranked unnecessary on the last day of life. Conversely the proportion of tests ranked neutral and necessary decreased over the seven day period (Figure 2). The interrater reliability for the specialists was found to be Kappa $=0.016(\mathrm{p}=0.06)$.

The highest proportion of unnecessary tests were found in neurologic (100\%) and haematologic $(79.45 \%)$ patients while gynaecologic (28.84\%) and head and neck $(34.40 \%)$ patients had the lowest. genitourinary $(11.62 \%)$ and lung $(11.84 \%)$ cancer patients had the highest proportion of necessary tests (Figure 3 ).

\section{Types of tests}

The most frequent test category ordered was blood chemistry

\section{Number of tests conducted per patient per day}

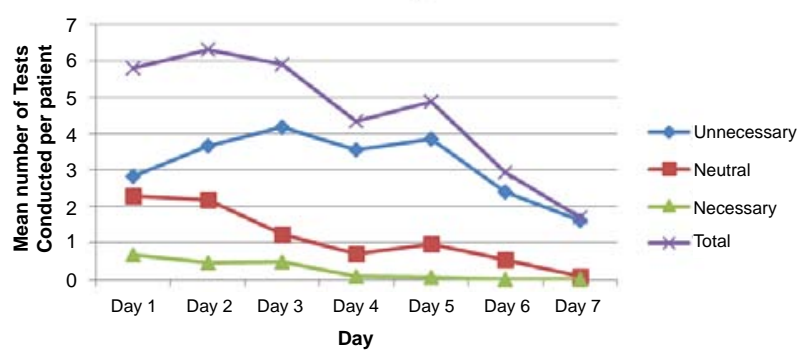

Figure 1: Number of tests conducted per patient per day.

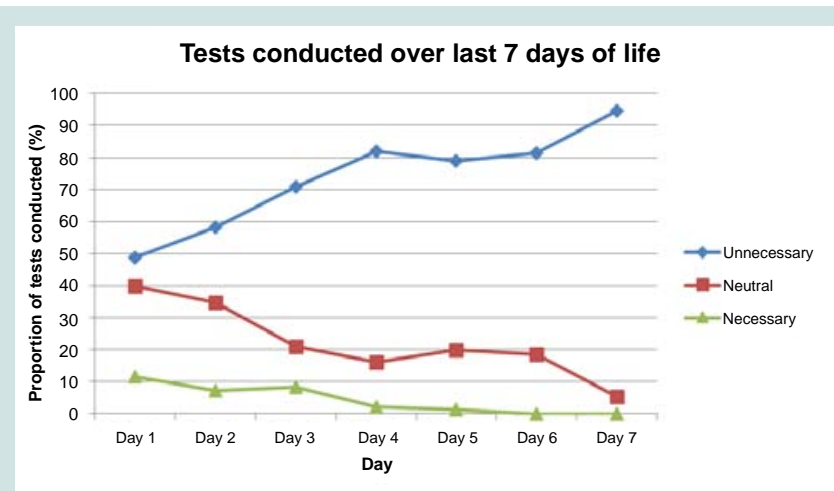

Figure 2: Tests conducted over last 7 days of life.

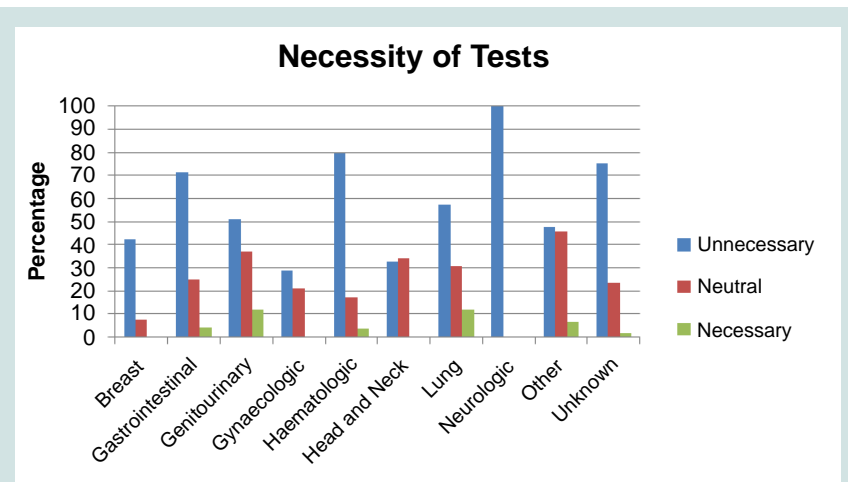

Figure 3: Necessity of tests by patient cancer type.
(41.19\%) followed by complete blood count haematology (28.83\%) and coagulation (10.47\%) tests. The highest percentage of Unnecessary tests were found in the tumour and immunology test categories while the nuclear medicine and microbiology test categories had the highest percentage of tests ranked as necessary. In total, $69.27 \%$ of all tests conducted were deemed unnecessary, 25.21\% neutral and 5.52\% necessary (Table 3 ).

\section{Discussion}

\section{Number and necessity of tests}

This study showed that the majority of tests being conducted in the last week of life were unnecessary. Invasive tests considered part of "routine" practice such as collecting blood can be a very traumatic experience for patients approaching end of life [7] and many of these could have been avoided. An earlier study conducted in one teaching hospital and one central hospital located in Finland from 1995 - 1998 [3] reported that the number of diagnostic tests conducted increased as death approached. However we found in our Australian teaching hospital setting there was an overall decrease in the number of tests being conducted over the seven day period as it became closer to death. Nevertheless, $21 \%$ of patients still had tests conducted on the very last day of life. Even more alarmingly, the proportion of tests that were unnecessary increased over the week prior to death, with $94.94 \%$ of tests conducted on the last day of life considered unnecessary. Our study found that $69.27 \%$ of the total tests conducted were deemed unnecessary, $25.21 \%$ neutral and $5.52 \%$ necessary. The proportion of unnecessary and neutral tests were significantly higher than those found in a general hospital in Switzerland by Sessa et al. [8] in 1995, who considered $33 \%$ unjustified, $15 \%$ optional and $50 \%$ necessary.

Laboratory tests account for a significant proportion of in hospital costs and many of these could be avoided. For example, in one of our cases a total of 45 tests were conducted in the last week of life. Of those three full blood counts, seven electrolyte and liver function tests and three calcium tests were deemed by our assessors as unnecessary. Using standard laboratory prices, these cost of these unnecessary tests amounted to a total of \$400AUD - expenditure that could have been saved by the healthcare system. A previous study showed that the hospital inpatient cost incurred for patients who died with cancer New South Wales, Australia is \$6073AUD per person in last 30 days of life [9]. Extrapolating from these results, $28 \%$ of in-patient costs in the last 7 days of life could be saved by not performing unnecessary tests during the end of life period for this patient. It is clear from this study that greater care should be taken by clinicians to recognise and acknowledge dying patients and actively minimise the number of unnecessary tests conducted in order to improve patient care. From the clinical record system, we realised that majority of blood tests ordered come from the junior medical officers of the teams. It is important for junior medical officers (intern and resident medical officers) be critical about the tests that they order in this particular situation instead of routinely doing the bloods for all patients admitted to hospital. Furthermore, registrars and consultant levels' medical officer should oversee the tests ordered for patients and advise their junior staff when one should stop doing any more blood tests.

\section{Types of tests}

Consistent with other studies [1,3], our study found that blood tests including blood chemistry and haematology constituted 
Citation: Rachel WC NG, Chi Eung Danforn LIM, Nga Chong Lisa CHENG, Rebecca STRUTT, Jennifer WILTSHIRE, et al. Number and Necessity of Tests Performed In the Last Week of Life of a Cancer Patient. J Geriatrics Palliative Care 2013;1(1): 5.

ISSN: 2373-1133

\begin{tabular}{|c|c|c|c|c|c|}
\hline Test Category & $\begin{array}{c}\text { Total Number (\% of } \\
\text { Total) }\end{array}$ & Number of Tests per Day & Unnecessary (\%) & Neutral (\%) & Necessary (\%) \\
\hline Blood Chemistry & $433(41.19)$ & 1.87 & 70.90 & 27.02 & 2.07 \\
\hline Haematology & $303(28.83)$ & 1.31 & 73.59 & 24.75 & 1.65 \\
\hline Coagulation & $110(10.47)$ & 0.48 & 71.82 & 17.27 & 10.91 \\
\hline Xray & $25(2.38)$ & 0.11 & 24.00 & 48.00 & 28.00 \\
\hline CT & $4(0.38)$ & 0.02 & 0.00 & 75.00 & 25.00 \\
\hline Nuclear Medicine & $1(0.10)$ & 0.00 & 0.00 & 0.00 & 100.00 \\
\hline Immunology & $108(10.28)$ & 0.47 & 90.74 & 9.26 & 0.00 \\
\hline Body Fluid & $8(0.76)$ & 0.03 & 25.00 & 75.00 & 0.00 \\
\hline Culture & $14(1.33)$ & 0.06 & 7.14 & 57.14 & 35.71 \\
\hline Microbiology & $9(0.86)$ & 0.04 & 0.00 & 11.11 & 88.89 \\
\hline Blood Gas & $16(1.52)$ & 0.07 & 37.50 & 37.50 & 25.00 \\
\hline Ultrasound & $3(0.29)$ & 0.01 & 0.00 & 66.67 & 33.33 \\
\hline Endocrinology & $6(0.57)$ & 0.03 & 50.00 & 33.33 & 16.67 \\
\hline Tumour & $2(0.19)$ & 0.01 & 100.00 & 0.00 & 0.00 \\
\hline Urine Analysis & $3(0.29)$ & 0.01 & 0.00 & 100.00 & 0.00 \\
\hline Cardiovascular & $1(0.10)$ & 0.00 & 0.00 & 100.00 & 0.00 \\
\hline Total & $1051(100)$ & 4.57 & 69.27 & 25.21 & 5.52 \\
\hline
\end{tabular}

the majority of investigations conducted. In our study $70.9 \%$ of blood chemistry and $73.59 \%$ of haematology tests were deemed inappropriate or unnecessary. Blood tests have become ingrained in the Australian hospital system as a "routine" procedure and results from our institution show that they are continued in the end of life period despite clear indication that the patient is dying and blood test results would have little or no effect on course of treatment or symptom management. Although it has been recommended that blood tests and vital sign measurements should be discontinued at the end of life stage to improve quality of physical care of patients [10], clinicians may find it difficult to explain to patients the reasons for discontinuing processes which patients have grown accustomed to $[11,12]$ and hence continue. Therefore it is probable that enhancing communication skills of clinicians would contribute to the quality of patient care in the last week of life.

Additionally, $100 \%$ of tumour marker level tests and $90.74 \%$ of immunology tests such as $\mathrm{C}$ reactive protein levels were found to be conducted unnecessarily. In patients with advanced stages of cancer, commonly performed tumour tests such as PSA levels and immunology tests do not identify any reversible causes of clinical deterioration or provide any benefit to the patient [11], yet were found to be performed regularly in the last week of life.

\section{Recommendation}

Advanced cancer patients who are recognised to be approaching end of life tend to receive inappropriate and inadequate care in an acute general hospital setting because their needs do not fit the conventional model and ideology of large hospitals where cure is seen as the main purpose of hospital care [7]. Our study has identified one aspect of end of life care that is frequently neglected: that of minimizing unnecessary tests. Greater education for clinicians needs to be implemented in regards to end of life care and recognising the patterns of dying patients with specific focus on identifying tests and interventions that are not necessary in the end of life period. Clear guidelines and indicators of clinical deterioration that is reversible, hence suitable for tests and interventions, should also be made available.

It has also been suggested that implementing flagging and warning systems on electronic medical record systems based on diagnosis and competing morbidities may also be used to warn doctors when tests are not necessary [11]. Another possible intervention would be to require that reasons for tests must also be inputted into electronic medical systems before orders can be made, to allow the opportunity for clinicians to evaluate the necessity of the test and benefit towards the patient.

Our results showed a negative correlation between total number of tests conducted and length of palliative care referral during their final admission (Days). This is consistent with earlier studies [13],which suggests that earlier referral and involvement of palliative care teams in managing patients would also help reduce the number of tests conducted and increase quality of patient care by reducing the unnecessary suffering that we may impose to patients who are terminally ill.

\section{Limitations of study}

Firstly, the small sample size of this study was one of the limitations of this study. Patients were only recruited in a single institution and over a short period of time and thus may not be an accurate representation of the wider population. Secondly, retrospective examination of clinical notes relies upon the quality of the documentation made by the doctors ordering the tests. Furthermore, discussions between various clinical staff, patient and family may not have been recorded. 
Citation: Rachel WC NG, Chi Eung Danforn LIM, Nga Chong Lisa CHENG, Rebecca STRUTT, Jennifer WILTSHIRE, et al. Number and Necessity of Tests Performed In the Last Week of Life of a Cancer Patient. J Geriatrics Palliative Care 2013;1(1): 5.

Additionally only data of the last seven days prior to death of the final admission was collected and therefore previous treatments, goals of care and other milestones were not included. These factors may have affected the specialist's ranking of necessity of tests conducted. Thirdly, retrospectively assessing the appropriateness of tests when the outcome is already known (death) is subject to bias. Rating of tests based on "necessity" is a subjective measure and requires further validation. Furthermore, the interrater reliability found in this study suggests only slight agreement. Including cases in which the patient did not die would minimize bias.

\section{Conclusion}

End of life cancer patients are subjected to frequent and often unnecessary tests and investigations. Much improvement can be made in hospital systems and clinician education in order to increase the quality of patient care. Our study recommends clinicians to evaluate the necessity and benefit of diagnostic tests conducted on end of life cancer patients before ordering them. Further training and education for clinicians in managing end of life patients is also recommended. Additionally, improved communication between clinician and patient and earlier referral to palliative care is also needed. Further research and development in the area of end of life care pathways, decision making tools for clinicians and increasing awareness of end of life issues could also help improve quality of care in terminal cancer patients.

\section{References}

1. Hui D, Con A, Christie G, Hawley PH (2009) Goals of Care and End-ofLife Decision Making for Hospitalized Patients at a Canadian Tertiary Care Cancer Center. J Pain Symptom Manage 38: 871-881.

2. Harrington SE, Smith TJ (2008) The Role of Chemotherapy at the End of Life : "when is enough, enough?". JAMA 299: 2667-2678.
3. Asola R, Huhtala $\mathrm{H}$, Holli K (2006) Intensity of diagnostic and treatment activities during the end of life of patients with advanced breast cancer Breast cancer research and treatment 100: 77-82.

4. Toscani F, Di Giulio P, Brunelli C, Miccinesi G, Laquintana D, et al. (2005) How People Die in Hospital General Wards: A Descriptive Study. J Pain Symptom Manage 30: 33-40.

5. Becker G, Sarhatlic R, Olschewski M, Xander C, Momm F, et al. (2007) Endof-Life Care in Hospital: Current Practice and Potentials for Improvement. J Pain Symptom Manage 33: 711-719.

6. Tse DM, Chan KS, Lam WM, Leu K, Lam PT (2007) The impact of palliative care on cancer deaths in Hong Kong: a retrospective study of 494 cancer deaths. Palliat Med 21: 425-433.

7. Middlewood S, Gardner G, Gardner A (2001) Dying in Hospital: Medical Failure or Natural Outcome? J Pain Symptom Manage 22: 1035-1041.

8. Sessa C, Pampallona S, Carobbio M, Neuenschwander H, Cavalli F (1998) Palliative care of cancer patients: audit of current hospital procedures. Support Care Cancer 6: 266-272.

9. Kardamanidis K, Lim K, Cunha CD, Taylor LK, Jorm LR (2007) Hospital costs of older people in New South Wales in the last year of life. Med J Aust 187: 383-386.

10. Ellershaw J, Ward C (2003) Care Of the Dying Patient: The Last Hours Or Days Of Life. BMJ 326: 30-34.

11. Sima CS, Panageas KS, Schrag D (2010) Cancer screening among patients with advanced cancer. JAMA 304: 1584-1591.

12. (1995) A Controlled Trial to Improve Care for Seriously III Hospitalized Patients. The study to understand prognoses and preferences for outcomes and risks of treatments (SUPPORT). The SUPPORT Principal Investigators. JAMA 274: 1591-1598.

13. Morrison R, Penrod JD, Cassel J, Caust-Ellenbogen M, Litke A, et al. (2008) Cost savings associated with us hospital palliative care consultation programs. Arch Intern Med 168: 1783-1790. 